[2] Yang H, et al. Int J Rheum Dis 2017.

[3] Chiba K, et al. Eular 2017.

Disclosure of Interest: None declared

DOI: 10.1136/annrheumdis-2018-eular.3851

\section{SAT0659 IS SYNOVIAL HYPERTROPHY WITHOUT DOPPLER ACTIVITY IN RHEUMATOID ARTHRITIS JOINTS SENSITIVE TO CHANGE? - RESULTS OF A LONGITUDINAL ULTRASOUND STUDY}

L. Terslev ${ }^{1}$, M. Østergaard ${ }^{1}$, J. Sexton ${ }^{2}$, H.B. Hammer ${ }^{2} .{ }^{1}$ Center for Rheumatology and Spine Disease, Rigshospitalet-Glostrup, Glostrup, Denmark; ${ }^{2}$ Rheumatology, Diakonhjemmet, Oslo, Norway

Background: Ultrasound (US) is used to assess diseases activity in rheumatoid arthritis (RA). Grey scale (GS) US shows the synovial hypertrophy (SH) and Doppler the amount of hyperemia which is believed to reflect disease activity. Some joints with SH may have no Doppler activity despite the use of high-end US equipment and these joints are generally believed to be inactive without potential to change.

Objectives: The aim was to investigate if joints with $\mathrm{SH}$ but no Doppler activity is sensitive to change during treatment with biological DMARD (bDMARD) in RA patients.

Methods: RA patients initiating or changing bDMARD treatment were included US examination was performed at baseline, 3 and 6 months using Siemens Antares US equipment with Doppler settings for slow flow.

36 joints were evaluated at each visit. SH and Doppler activity was graded from 0-3 according to the US atlas by Hammer et al. $\left({ }^{1}\right.$ The GS score for $\mathrm{SH}$ in joints without Doppler activity was registered for the individual joints using $\mathrm{GS} \mathrm{SH} \geq 1$ as threshold. The changes were compared to changes in $\mathrm{SH}$ in joints with Doppler activity.

Results: 151 patients ( $82.8 \%$ women, $80.1 \%$ seropositive for anti-CCP) were included, with a mean (SD) age 51.4 (13.2) years, disease duration 9.9 (7.9) years. At baseline, 50.7\% used prednisolone (mean (SD) 5 mg (4.68)).

The patients had a mean (SD) baseline DAS28 of 4.5 (1.5). At baseline $23 \%$ of the joints had SH without Doppler activity and $23 \%$ of the joints had SH with Doppler activity.

Doppler-negative joints had overall lower grades of SH (mean 1.2) than Dopplerpositive joints (mean 2.2) at baseline using GS SH $>1$ as cut-off. The improvement in SH was similar in Doppler-positive and Doppler-negative joints but when adjusting for the baseline score of SH, Doppler-negative joints had a higher tendency towards decrease than Doppler-positive joints for all grades (3 months: $p<0.0001$; 6 months $=0.0006$ )

A weak correlation was found to changes in DAS 28(crp) (Doppler-negative joints:0.27 $(p=0.001)$ and $0.18(p=0.03)$ at 3 and 6 months respectively - Dopplerpositive joints: $0.25(p=0.004)$ and $0.33(p=0.0002)$ at 3 and 6 months respectively).

Conclusions: $\mathrm{SH}$ in joints without Doppler activity improves during bDMARD, i.e is sensitive to change. Thus, $\mathrm{SH}$ without Doppler activity is not a sign of inactive disease. These findings document that both Doppler and SH should be evaluated when assessing disease activity by US

\section{REFERENCE:}

[1] Hammer HB, Bolton-King P, Bakkeheim V, et al. Examination of intra and interrater reliability with a new ultrasonographic reference atlas for scoring of synovitis in patients with rheumatoid arthritis. Ann Rheum Dis 2011;70:1995-8.

Disclosure of Interest: None declared

DOI: 10.1136/annrheumdis-2018-eular.2265

\section{SAT0660 CLINICAL SIGNIFICANCE OF ANTI-RA33 AUTOANTYBODIES IN RHEUMATOID ARTHRITIS}

\section{Volkava, A. Kundzer. BelMAPGE, Minsk, Belarus}

Background: Recently heterogeneous nuclear ribonucleoproteins (hnRNPs) were identified as antigenic targets for autoantibodies in RA. Autoantibodies to RNP B1, B2 and heterogeneous complex A2/b are the most studied and are referred to as "antigen RA33". Clinical value of anti-RA33 in RA continues to be studied, so the question is about the diagnostic and pathogenetic significance of these autoantibodies in patients with RA remains open.

Objectives: The aim of this study was to investigate the frequency of occurrence of anti - RA33y patients with RA and compare them with clinical manifestations and immunological parameters of disease.
Methods: The study included 139 patients with RA. The diagnosis was verified according to the classification criteria of ACR/. ${ }^{\text {EULAR } 2010}$ Autoantibodies to RNP B1 lgG was assessed in samples of blood serum by enzyme immunoassay (ELISA; Medipan AG, Germany). Anti-CCP, RF and antibodies to Sa-antigen (anti-Sa) was evaluated by ELISA according to the manufacturer's instructions (Euroimmun AG company,Germany). The results were expressed in relative units $(\mathrm{U} / \mathrm{ml})$. Statistical data processing was carried out using the software Statistica 7.0 (StatSoft, USA) and Medcalc 12.5.0.0 (USA), including standard methods of parametric and nonparametric analysis. Differences were considered significant at $p<0,05$.

Results: In our cohort of patients with RA, the incidence rate of RF IgM was $96.02 \%$, anti-CCP $-81.75 \%$, anti-Sa $-69.88 \%$, anti-RA33\%-35.97\% of patients For further analysis of the clinical significance of anti-RA33 patients were divided into 2 groups: anti-RA33 positive and anti-RA33negative

Positive and negative for anti-RA33 patients did not differ ( $p>0.05)$ by key characteristics, such as age, gender, duration of disease, and indicators of disease activ ity (CRP levels, DAS28, SDAI, CDAI). In contrast to the data obtained Maslyansky A. L. and co-authors the correlation relationship between the levels of anti-RA33 and indicators of disease activity as well as duration of disease has not been established. At the same time seronegative for anti-RA33 patients demonstrated a higher frequency of exrtaarticular manifestations $(p<0,05)$.

When analysing the frequency of occurrence of anti-CCP, RF IgM, anti-Sa and anti-RA33 autoantibodies, there were no differences between the groups of sero positive and seronegative for anti-RA33. Correlation relationship between the levels of the investigated autoantibodies is not established.

Conclusions: Thus anti-RA33 is a perspective independent biomarker of RA which has its own potential. The possible pathogenic significance of anti-RA33 as a biomarker of a more favourable course of the RA should be considered.

To clarify the diagnostic and pathogenic value of anti-RA33 further research with a large sample size, comparison of immunological data with genetic factors, the results of other laboratory and instrumental studies, clinical manifestations of the disease are required. The obtained data will allow us to more thoroughly study the pathogenesis of RA and contribute to the search for new therapeutic options.

Disclosure of Interest: None declared

DOI: 10.1136/annrheumdis-2018-eular.6873

\section{SAT0661 MEASUREMENT OF THE INTIMA MEDIA THICKNESS OF TEMPORAL AND AXILLARY ARTERIES IN SUBJECTS WITH DIFFERENT RISKS OF CARDIOVASCULAR DISEASE: AN ULTRASOUND STUDY}

M.V. Martire ${ }^{1}$, A. Di Matteo ${ }^{2}$, E. Filippucci ${ }^{2}$, E. Cipolletta ${ }^{2}$, D. Jesus ${ }^{3}$, M. Di Carlo ${ }^{2}$, W. Grassi ${ }^{2}$. ${ }^{1}$ Instituto Médico Platense, La Plata, Argentina; ${ }^{2}$ Clinica Reumatologica, Università Politecnica delle Marche, Jesi (Ancona), Italy; ${ }^{3}$ Centro Hospitalar e Universitario de Coimbra, Coimbra, Portugal

Background: The "halo sign" is considered the well known qualitative ultrasound (US) finding for the diagnosis of giant cell arteritis (GCA). Recently, the measurement of the intima-media thickness (IMT) of temporal and axillary arteries has been proposed as a potential complementary US biomarker. A cut-off for IMT of $0.42,0.29,0.34$ and $1.0 \mathrm{~mm}$ for superficial temporal arteries, parietal and frontal branches and axillary arteries, respectively, has been suggested. In fact, other conditions, such as atherosclerosis, may determine an increase of the IMT.

Objectives: To measure the IMT of the temporal and axillary arteries in subjects with different risks of cardiovascular $(\mathrm{CV})$ disease and to investigate the prevalence of IMT values greater than the reference cut-off values.

Methods: Consecutive patients older than 50 years without signs or symptoms and without a previous history of GCA or polymyalgia rheumatica, were included. The European Guidelines of CV disease prevention were used to define different categories of CV risk. The subjects were divided in two groups: a first group made up of subjects with very high or high risk of CV disease and a second group made up of subjects with moderate or low risk of CV disease. The US examination was performed with a My Lab Twice (Esaote S.p.A. Genoa, Italy). The tempora arteries (superficial temporal arteries, parietal and frontal branches) were evaluated using a 18-22 $\mathrm{MHz}$ probe, whereas the axillary arteries were studied using a 6-18 MHz probe.

Results: Eight hundred and eight arteries were evaluated in 101 subjects (mean age 66.1 SD $8.5,73.3 \%$ females). Thirty-one subjects $(30.7 \%)$ were classified with very high risk of CV disease, 7 (6.9\%) with high risk, $34(33.7 \%)$ with moderate risk and $29(28.7 \%)$ with low risk. The IMT of the superficial temporal and axillary arteries were significantly higher in the group made up of subjects with very high or high risk of CV disease than in the group made up of by subjects with moderate or low risk of CV disease (0.21 SD 0.82 vs 0.19 SD $0.86 p$ :0.013; 0.54 SD 0.17 vs 0.48 SD0.10 p:0.20). The value of the IMT was higher than the reference cut-off in 13 out of $808(1.61 \%)$ of the studied arteries (2 superficial temporal artery, 6 parietal branch, 4 frontal branch and 1 axillary artery), in at least one artery in 10 out 101 patients $(10.1 \%)$. Of these 10 patients, $8(98 \%)$ were classified as having very high or high risk of $\mathrm{CV}$ disease. 